\title{
Neuronal Lamin regulates motor circuit integrity and controls motor function and lifespan
}

\author{
Lisa J. Oyston ${ }^{1,2,3, *}$, Yong Qi Lin ${ }^{1}$, Thang M. Khuong ${ }^{1}$, Qiao-Ping Wang ${ }^{1}$, Man Tat Lau ${ }^{1}$, Teleri Clark $^{1}$ and G. \\ Gregory Neely ${ }^{1, *}$ \\ ${ }^{1}$ The Dr. John and Anne Chong Lab for Functional Genomics, Charles Perkins Centre and School of Life and Environmental Sciences, \\ The University of Sydney NSW 2006, Australia. \\ ${ }^{2}$ Neuroscience Division, Garvan Institute of Medical Research, 384 Victoria Street, Darlinghurst, Sydney, NSW 2010, Australia. \\ ${ }^{3}$ St Vincent's Clinical School, Faculty of Medicine, UNSW Sydney NSW 2052, Australia. \\ * Corresponding Authors: \\ G. Gregory Neely, T: +61 28627 0519; E-mail: greg.neely@sydney.edu.au; \\ Lisa Oyston, T: +61 28627 5061; E-mail: lisa.oyston@sydney.edu.au
}

\begin{abstract}
Neuronal aging involves a progressive decline in cognitive abilities and loss of motor function. Mutations in human Lamin genes (LMNA, LMNB1, LMNB2) lead to a wide-range of diseases including muscular dystrophy, peripheral neuropathy and progeria. Here we investigate the role of neuronal Lamin in regulating age-related phenotypes. Neuronal targeting of Lamin led to shortened lifespan, progressive impairment of motor function and loss of dopaminergic (DA) neurons within the protocerebral anterior medial (PAM) cluster in the Drosophila melanogaster brain. Loss of neuronal Lamin caused an age-related decline in neural physiology, with slower neurotransmission and increased chance of motor circuit failure with age. Unexpectedly, Lamindependent decline in motor function was specific for the chemical synapses of the dorsal longitudinal muscle (DLM). Together these findings highlight a central role for Lamin dysfunction in regulating neuronal survival and motor circuit physiology during aging.
\end{abstract}

doi: $10.15698 /$ cst2018.09.152

Received originally: 15.05.2018; In revised form: 26.07.2018,

Accepted 30.07.2018,

Published 17.08.2018.

Keywords: aging, Lamin, dopaminergic neurons, neurodegeneration, Drosophila, synapse.
Abbreviations:
$D A$-dopaminergic,
$D L M$ - dorsal longitudinal muscle,
GFS - giant fiber system,
PAM - protocerebral anterior medial,
TTM - tergotrochanteral muscle.

\section{INTRODUCTION}

Nuclear architecture and nuclear membrane function is a critical regulator of the aging process [1]. The nuclear lamina is a filamentous network that lines the inner nuclear membrane, providing a structural scaffold for the nucleus. This network is made up of type $\mathrm{V}$ intermediate filaments known as lamins that tether protein and chromatin complexes to the inner nuclear membrane. Mammals possess three lamin genes, an A-type gene, LMNA which encodes the alternatively spliced variants lamin $A$ and lamin $C$, as well as two B-type genes, LMNB1 and LMNB2 [2]. Mutations in human Lamin genes (LMNA, LMNB1, LMNB2) cause a range of severe disorders termed laminopathies, which include muscular dystrophies, peripheral neuropathies and progeria, an accelerated aging syndrome [2]. Alterations in the nuclear lamina have also been implicated in normal aging, suggesting that nuclear integrity and genomic stability are critical for cellular health throughout life [3].
B-type lamins are constitutively and ubiquitously expressed whilst A-type lamins show more specific expression patterns in differentiated cells [4]. Differential expression patterns of A- and B-type lamins may explain the tissue-specific pathology that occurs in many laminopathies, for example, the absence of dementia in HutchinsonGilford progeria patients, which is caused by mutations in LMNA.

Drosophila are the only invertebrates known to possess both A (Lamin C) and B-type lamins (Lamin Dm0; referred to as Lamin throughout this publication) [5]. Both Drosophila and vertebrate lamins evolved from a single gene in a common ancestor and possess similar expression patterns and molecular motifs $[5,6]$.

The role of the nuclear lamina within the adult brain is somewhat unclear. Lamin is the only nuclear lamin found to be expressed in the nervous system $[7,8]$. Even in Lamin null mutant flies, Lamin $C$ is not detectable, suggesting limited compensation within this compartment $[7,8]$. B- 
type Lamin genes (Lmnb1, Lmnb2) are essential for neuronal survival and migration [9], while Lamin expression decreases in the aging Drosophila brain [10]. Moreover, transgenic expression of Tau causes a decrease in lamin protein, and a Lamin partial loss of function mutation significantly reduces lifespan and promotes neuronal apoptosis [11]. Here, we investigate the role of Lamin on neurological function within the aging Drosophila brain.

\section{RESULTS}

To specifically assess the role of Lamin within the nervous system, we used a tissue-specific transgenic RNAi approach [12]. elavGal4 drives transgenic GFP expression (UAS$m C D 8 G F P$ ) across the fly brain (Fig S1A-F) and these flies showed no difference in lifespan or motor function when compared to elavGal4 alone (Fig S1G-H). Neuronal knockdown of Lamin with two independent RNAi lines showed a significant reduction in lifespan (Fig 1A, Fig S1I-K). For each RNAi line, neuronal knockdown of Lamin was $\sim 50 \%$ of control levels by qPCR (Fig 1B), and western blot of heads from elav/Lam IR flies also showed a $\sim 60 \%$ reduction in Lamin protein levels (Fig 1C). Functionally, a decrease in neuronal Lamin led to a rapid and progressive loss of motor function over the first 21 days of life (Fig 1D) and overall, neuronal Lamin caused an $\sim 80 \%$ decline in climbing ability over the fly's lifespan (Fig 1E). Taken together, decreased expression of Lamin within the nervous system is sufficient to shorten lifespan and impair motor function with age.

DA neurons are known to be responsible for locomotor decision making and fine tuning in vertebrates and invertebrates [13] and are well characterised within the fly brain $[14,15]$. Decline in motor function has also been associated with progressive loss of DA neurons with age [16]. Moreover, in Drosophila, it has been shown that neurons
A

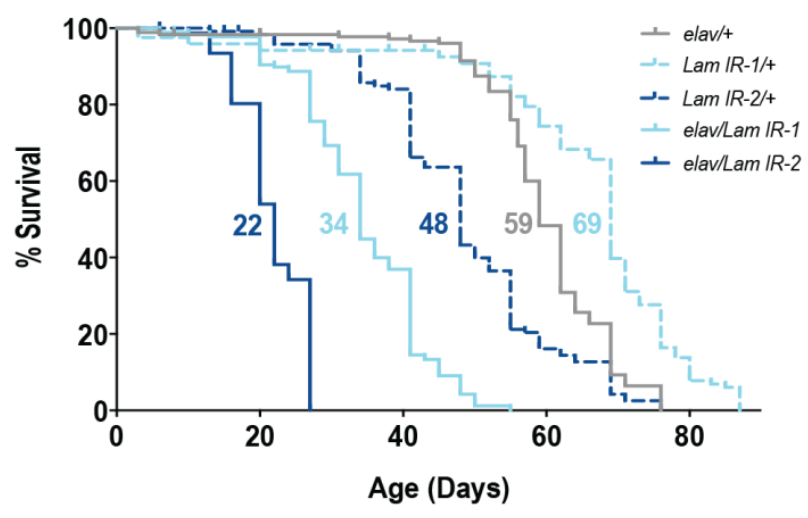

D

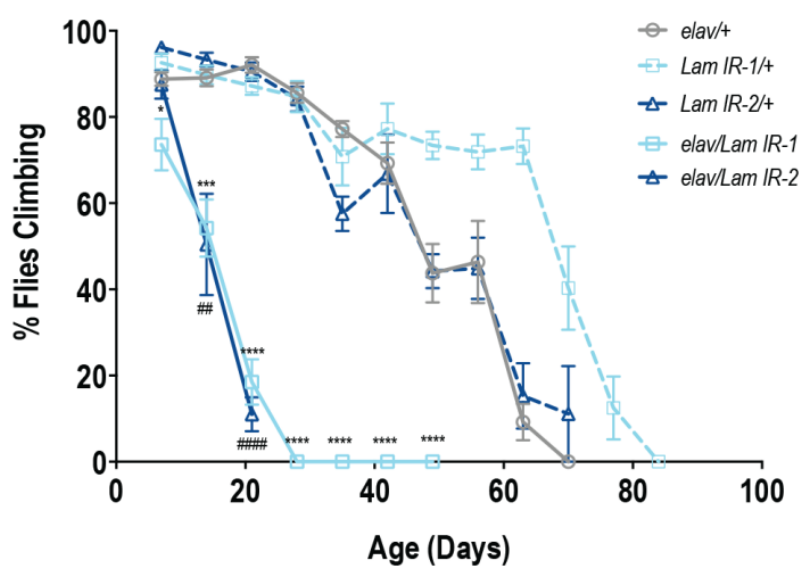

B

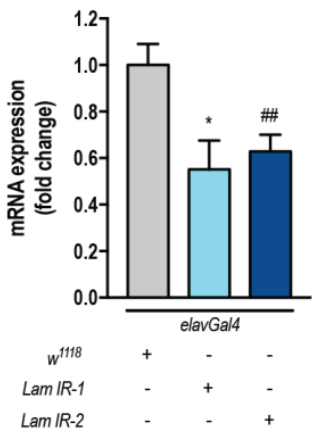

C
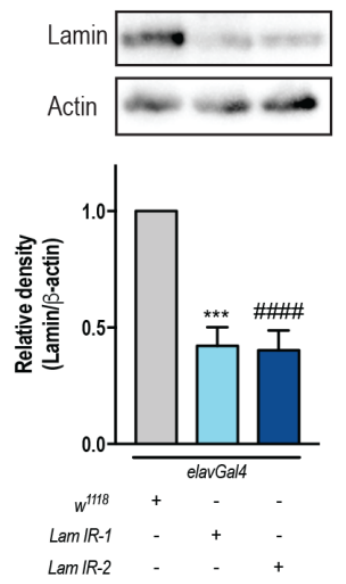

E

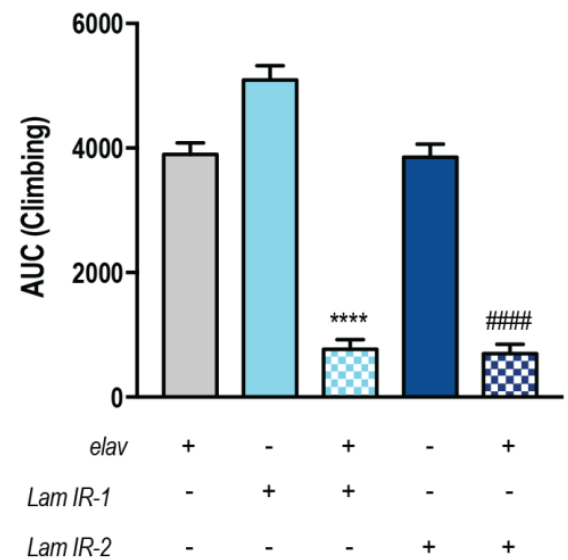

FIGURE 1: Neuronal knockdown of Lamin causes decreased lifespan and locomotor ability. (A) Pan neuronal knockdown of Lamin (elav/Lam IR) causes a significant decrease in lifespan compared to control flies (elav/+). (B) qPCR for knockdown efficiency of Lamin RNAi hairpins showed a significant decrease in Lamin mRNA expression for both hairpins. (C) Western blot of fly heads showed a significant decrease in Lamin protein expression with Lam IR-1 and Lam IR-2 knockdown. $\mathrm{n}=5$. (D-E) Locomotor ability is significantly impaired in elav/Lam IR flies. $\mathrm{n} \geq 50$ animals per group. Data represents mean \pm SEM. Climbing, western blot and qRT-PCR data was analysed using oneway ANOVA with post-hoc Dunnett test for multiple comparisons. Lifespan analysis was done using a log-rank (Mantel-Cox test). ${ }^{*}$ comparison between elav/+ and elav/Lam IR-1. \#comparison between elav/+ and elav/Lam IR-2. ${ }^{*} \mathrm{p}<0.05 ;{ }^{* *} \mathrm{p}<0.01 ;{ }^{* * *} \mathrm{p}<0.001 ;$ $* * * * p<0.0001$. elav/+ = elavGal4; +/+; UAS-mCD8GFP $>w^{1118} ;$ Lam IR/+ = $w^{1118}>$ Lam IR; elav/Lam IR = elavGal4; +/+; UAS-mCD8GFP $>$ Lam IR. 
within the PAM cluster on the anterior side of the fly brain are responsible for startle-induced locomotor function (Fig 2D) [17]. Neuronal Lamin knockdown animals displayed no apparent loss of DA neurons across most areas of the brain (Fig 2A-C, G, S2A-D, I), however we did observe a progressive loss of DA neurons within the PAM cluster (Fig 2D-F, S2E-H), and this effect was most pronounced in aged animals (Fig 2H). Assessment of DA neurons in 50-day old elav/Lam IR-2 flies was not possible due to their maximum lifespan of 27 days (Fig 1A). However, loss of PAM DA neurons was observed in elav/Lam IR-2 flies at 10 days of age
(Fig $\mathbf{2 H}$ ), suggesting fly lifespan may be correlated to DA neuron loss in the PAM cluster. Together our results suggest that neuronal Lamin levels are critical for the brain during aging, and dysregulation of Lamin expression can lead to degeneration of DA neurons.

Due to the profound effects of neuronal-specific Lamin knockdown on age-related behaviours (climbing, lifespan and neurodegeneration), we decided to investigate its effects on neuronal function. Age-related deficits in Drosophila lifespan and climbing behaviors manifest in the functional decline of the fly's giant fiber system (GFS) [18], a elav/t+

A

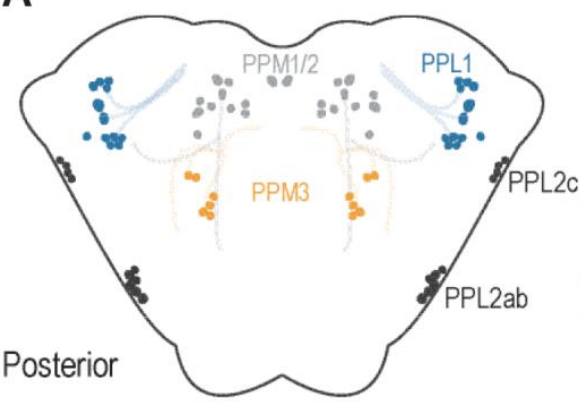

D

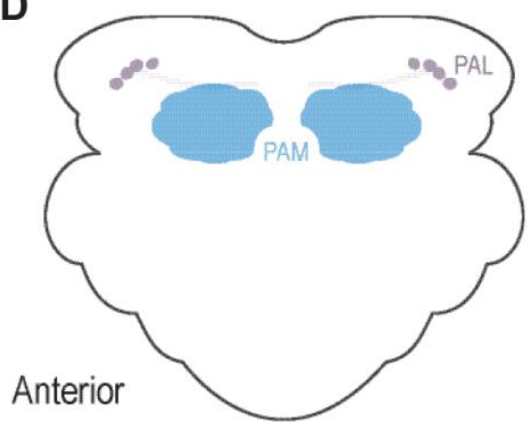

G

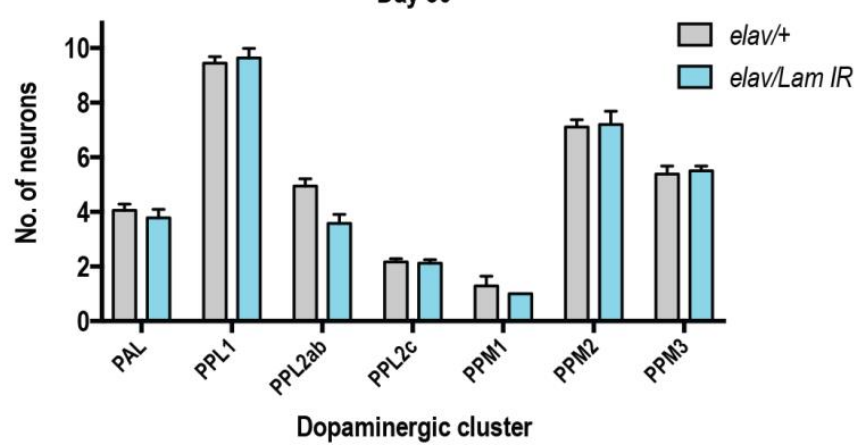

Day 50
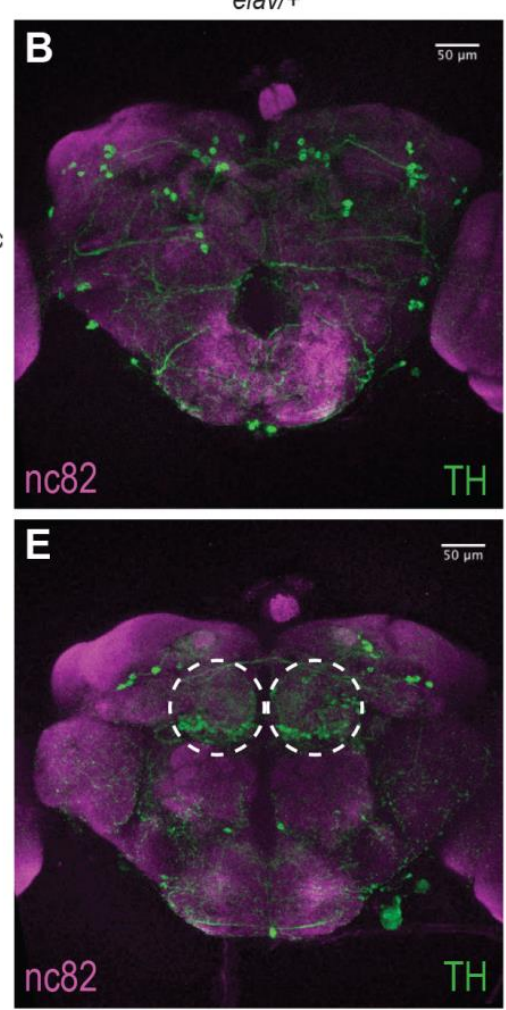

H
lav/Lam IR
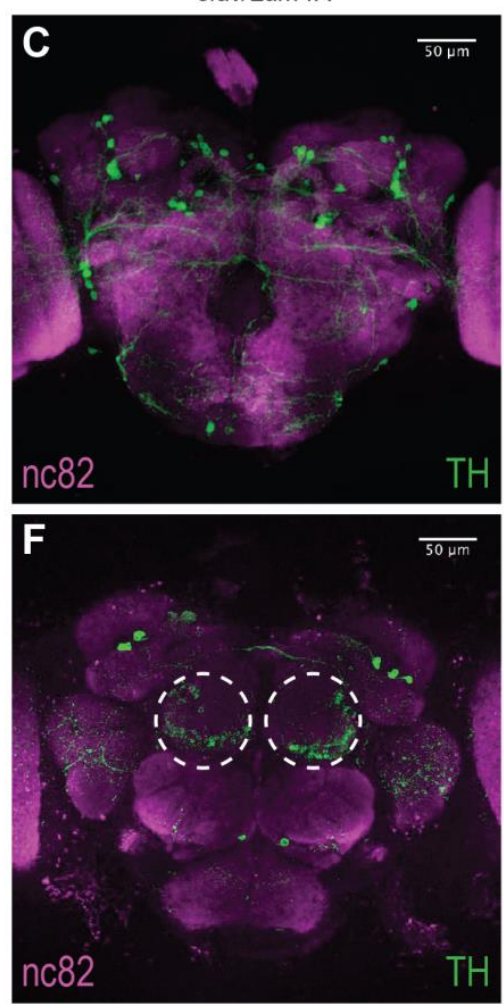

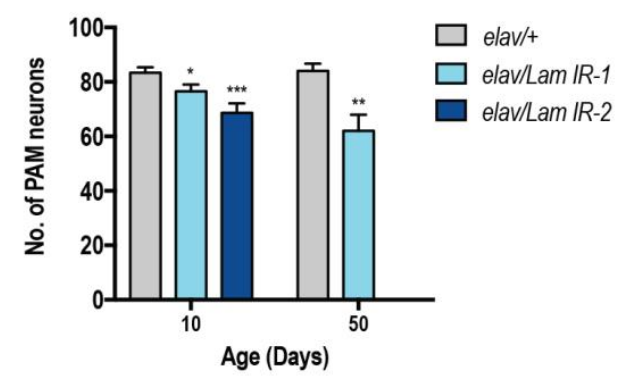

FIGURE 2: Neuronal knockdown of Lamin causes an age-dependent loss of PAM cluster dopaminergic neurons. (A) Schematic of the posterior dopaminergic neurons of the Drosophila brain. Representative images of posterior dopaminergic neuron clusters in (B) elav/+ and (C) elav/Lam IR flies at 50 days old. (D) Schematic of the anterior dopaminergic neurons of the Drosophila brain. Representative images of the anterior dopaminergic neuron clusters in (E) elav/+ and (F) elav/Lam IR flies at 50 days old. G Quantification of dopaminergic neuron number in the posterior and anterior clusters of the fly brain at 50 days. (H) Quantification of dopaminergic neurons within the PAM cluster of the fly brain. $\mathrm{n} \geq 7$ animals per group. Data represents mean \pm SEM and was analysed using student's $t$-test. ${ }^{*} p<0.05$; ${ }^{* *} \mathrm{p}<0.01 ;{ }^{* * *} \mathrm{p}<0.001$. elav/+ = elavGal4; +/+;UAS-mCD8GFP $>w^{1118} ;$ elav/Lam IR = elavGal4; +/+; UAS-mCD8GFP $>$ Lam IR. 
well-defined neuronal circuit that controls jump and flight responses $[19,20]$.

To assess neurotransmission in the GFS, we recorded from the DLM, the indirect flight muscle which is controlled via a glutamatergic synapse from the peripherally synapsing interneuron (PSI; Fig 3A) [20]. Compared to control animals, Lamin knockdown animals showed a progressive loss of circuit function, manifesting in response failures on repeated train stimulation (Fig 3B-C). Quantification of these results highlights the loss of motor circuit function over time (Fig 3D-F) with maximal failures (11.32 \pm 2.57 ) observed in 50-day old flies with neuronal Lamin knockdown at $150 \mathrm{~Hz}$ (Fig 3F). Control flies across all ages and all stimulation frequencies showed $<1$ mean failures (Fig 3D-F). Qualitatively, the probability of response for Lamin knockdown animals declined on repeated stimulation at each age point (Fig 3G-I), however, 50-day old animals showed baseline defects in circuit responses even without repeated stimulation, highlighting the extreme age-related loss of motor circuit function in these flies. No difference was observed in DLM response latency at 10 days in Lamin knockdown or control flies (Fig 3J). Moreover, DLM response latency was significantly increased in Lamin knockdown flies at 30 days of age (Fig $\mathbf{3 K}$ ) compared to control. This effect could arise from direct loss of circuit or neuromuscular junction integrity, or indirectly via trans-synaptic effects that alter the responsiveness of the post-synaptic muscle. A significant difference in latency was also observed at 50 days and $100 \mathrm{~Hz}$ stimulation, however due to the $70-75 \%$ failure rate at the $20^{\text {th }}$ pulse in 50 -day-old Lamin knockdown flies stimulated at $150 \mathrm{~Hz}$, only a few latency values could be calculated and no significant difference was observed in this case (Fig 3L). Together, we show that neuronal-specific loss of Lamin triggers a severe agerelated decline in motor circuit performance.

Recordings were also taken from the tergotrochanteral muscle (TTM), which controls the "jump" phase of the fly's escape response via mixed electrochemical synapses [20]. Unexpectedly, no significant increase in failure rate (Fig S3A-E), decrease in response probability (Fig $\mathbf{3 M - O}$ ), or change in circuit latency (Fig S3F-H) was observed. Together, these data show that DLM synapses are sensitive to changes in the nuclear lamina.

\section{DISCUSSION}

Drosophila has been an essential model organism in the discovery of the plasticity of aging, helping to identify genetic pathways and biochemical processes capable of influencing the rate of aging $[21,22]$. Here we expand on previous findings [11], to show targeting neuronal Lamin causes a progressive loss of locomotor function and decreased lifespan. These results are consistent with previous data on Lamin null and partial loss of function mutants that have been shown to be lethal or semi-lethal with surviving adults experiencing locomotor deficits and decreased lifespan [7, 8, 11, 23]. Studies of neuronal Lamin knockdown previously conducted by Frost et al. (2016) also showed comparable locomotor dysfunction to our study in the absence of decreased lifespan [11]. This discrepancy may be due to differences in genetic background, diet or environment and further investigation is required.

Defects in negative geotaxis behaviour have previously been accompanied by age-dependent loss or decreased tyrosine hydroxylase immunoreactivity in Drosophila DA neurons [24-28]. Here we show that neuronal Lamin knockdown is sufficient to cause age-dependent loss of DA neurons within the PAM cluster, however, the mechanism behind this loss remains unclear. Lamin dysfunction has previously been shown to cause apoptotic neuron death as a result of genomic architecture disruption, DNA damage and cell-cycle reactivation [11]. Thus, selective DA neurodegeneration in Lamin knockdown flies may occur due to increased susceptibility of PAM cluster neurons to age-related disruptions in nuclear envelope integrity, ultimately resulting in apoptosis. Behaviourally, DA neurodegeneration may then lead to decreased locomotor function and shortened lifespan.

Of note, we observed enhanced survival and climbing in Lam IR-1/+ lines when compared to the elav/+ or Lam $I R-2 /+$ control groups. Based on these results, we cannot determine if $L a m I R-1 /+$ flies exhibit enhanced health or if elav/+ and Lam IR-2/+ animals show a relative decrease in lifespan and motor function. Factors that could mediate this effect include an overall weakness of elav/+ animals due to gal4 expression, or a non-specific leakiness of the Lam IR-2 transgene which confers a disadvantage to this line. Alternatively, as these lines have been created a number of years ago, it is possible that secondary unknown mutations affect the health of one or more lines. Regardless, elav/Lam IR animals showed a 50\% reduction in lifespan compared to either parental control consistent with a specific role for Lamin in promoting neuronal health.

In this study, we have shown an age-dependent decrease in conduction velocity and an elevated failure rate at the Drosophila DLM following decreased Lamin expression. Martinez et al. (2007) have previously shown the effects of aging on neuronal function within the GFS, showing an association between aging and the number of successful DLM responses to high frequency stimulation in wild-type flies (100-200 Hz). In their study, elevated failure rate of the DLM muscle occurred in 35-45 days old animals stimulated above $150 \mathrm{~Hz}$ [18]. In our study, we did not observe failures in 50-day old wild type animals, however our maximum stimulation was $150 \mathrm{~Hz}$, and thus a lack of failure at this frequency is consistent with the findings of Martinez et al. (2007). We have shown a progressive increase in DLM failure rate in Lamin knockdown flies starting at day 10 and these animals exhibit dramatically reduced lifespan. The synaptic impairment of the DLM response and shortened lifespan induced by decreased Lamin expression are reminiscent of Drosophila models of motor neuron disease $[29,30]$, suggesting that neurodegeneration may be responsible for the phenotypes observed here.

We also observed that TTM latency and failure rate were unaffected by Lamin knockdown. The DLMn is pre- 
A

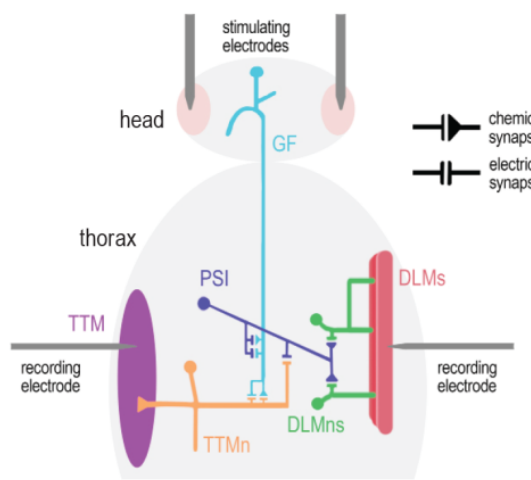

D

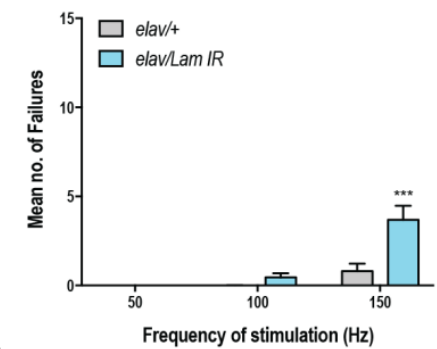

G

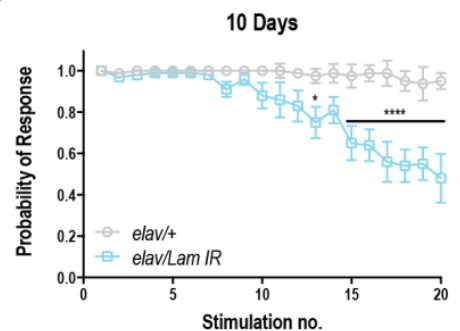

J

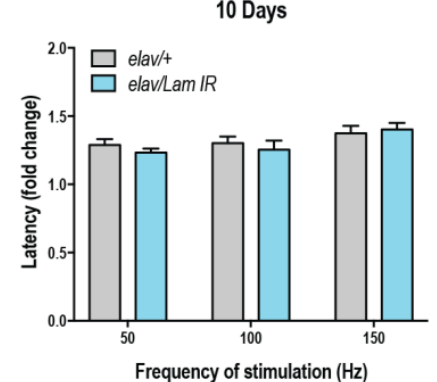

M

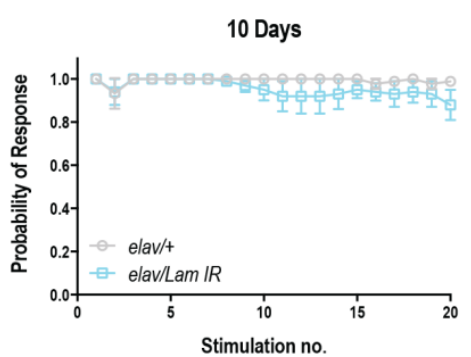

E

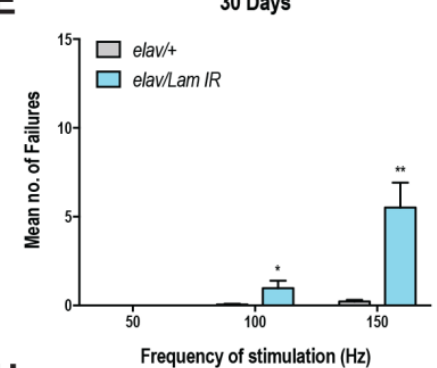

H

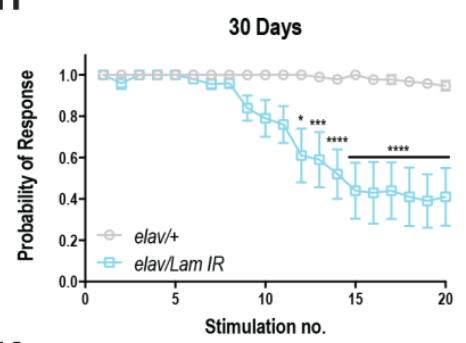

K

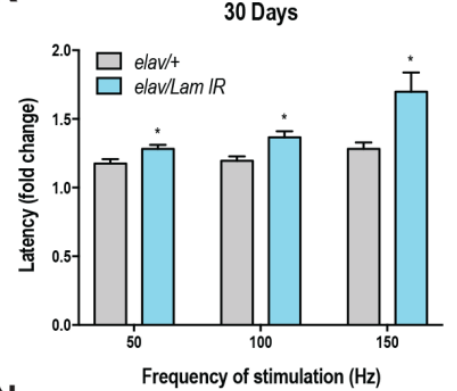

N

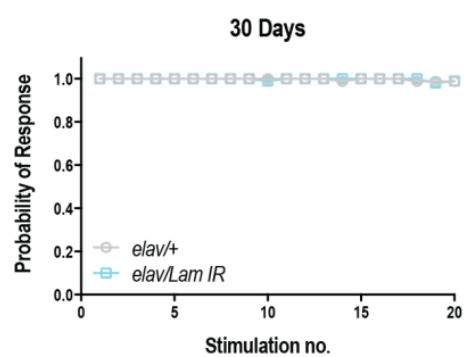

B

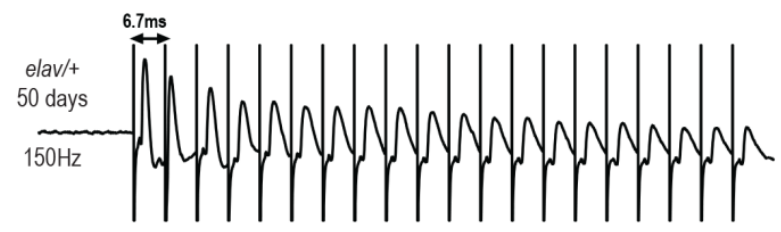

C

* failure

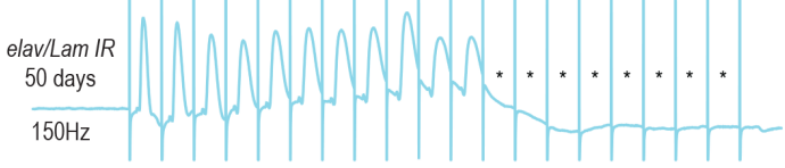

F

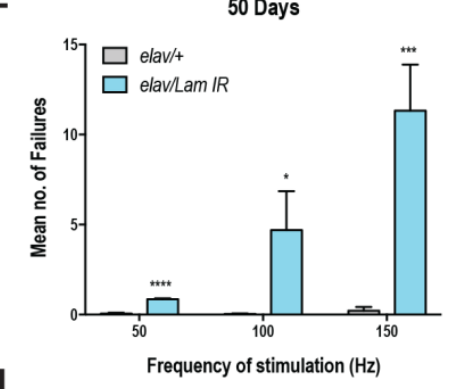

50 Days

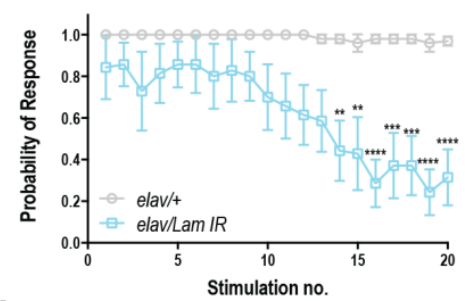

L

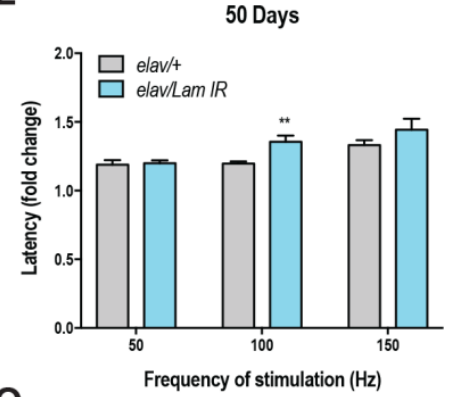

0

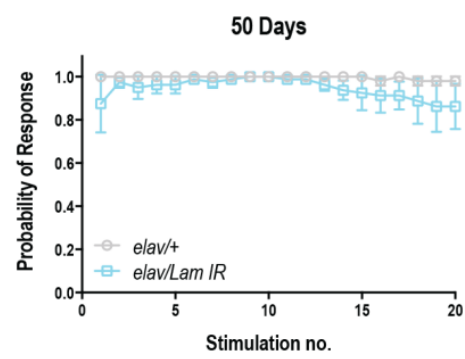

FIGURE: 3 Neuronal knockdown of Lamin causes increased failure and latency of the DLM. (A) Schematic of experimental setup for recording from the GFS. TTM = tergotrochanteral muscle; TTMn = tergotrochanteral motorneuron; PSI= peripherally synapsing interneuron; DLM = dorsal longitudinal muscle; DLMNn = dorsal longitudinal motorneuron. Representative traces from the DLM in (B) elav/+ and (C) Lamin knockdown flies (elav/Lam IR) aged 50 days, stimulated at $150 \mathrm{~Hz}$. Asterisks indicate failure to respond. Mean number of failures for elav/+ and Lamin knockdown flies (elav/Lam IR) at (D) 10 days, (E) 30 days and (F) 50 days old. (G-I) The probability of DLM response in Lamin knockdown flies (elav/Lam IR) is significantly decreased after repeated stimulations, in an agedependent manner. $(\mathrm{J})$ No significant difference in latency was found in young flies (10 days) with Lamin knockdown (elav/Lam IR) when compared to elav/+. (K-L) Aged flies (30 days and 50 days) show a significant increase in DLM latency with Lamin knockdown (elav/Lam IR). (M-O) The probability of TTM response in Lamin knockdown flies (elav/Lam IR) shows no significant difference from elav/t. $\mathrm{n} \geq 7$ animals per group. Data are mean \pm SEM. Data was analysed using two-way ANOVA with Bonferroni test for multiple comparisons. Response latency was analysed using multiple t-tests with Holdam-Sidak correction for multiple comparisons. ${ }^{*} \mathrm{p}<0.05 ;{ }^{* *} \mathrm{p}<0.01 ;{ }^{* * *} \mathrm{p}<0.001 ;{ }^{* * *} \mathrm{p}<0.0001$. elav $/+=$ elavGal4; $+/+;$ UAS-mCD8GFP $>w^{1118} ;$ elav/Lam IR $=$ elavGal4; +/+; UAS-mCD8GFP> Lam IR. 
dominantly activated by chemical glutamatergic synapses; the TTMn on the other hand is predominantly activated by electrical gap junctions directly from the GF [20]. The differences in response between the compound action potentials could thus be explained by the fact that Lamin defects are specific to chemical synapses rather than electrical gap junctions [31]. Alternatively, RNAi efficiency may differ between TTM and DLM circuits, which could explain why TTM responses were independent of Lamin. Neuromuscular junction defects have also been reported in Drosophila Lamin $C$ mutants, caused by disruptions to nuclear budding leading to improper differentiation of synaptic boutons [32]. Together these results suggest that Lamin dysfunction at neuromuscular junctions may be behind the manifestation of some laminopathies (muscular dystrophies) and Parkinson's disease?-like phenotypes.

Overall, we show here that neuronal-specific Lamin is a critical regulator of lifespan, neurodegeneration, and motor circuit health, and dysregulation of neuronal Lamin expression/function may contribute to multiple pathologies associated with aging and age-related diseases.

\section{MATERIALS AND METHODS \\ Fly stocks}

Flies were maintained on a standard diet of agar, corn meal, yeast and molasses at $25^{\circ} \mathrm{C}, 65 \%$ humidity under a $12 \mathrm{~h}: 12 \mathrm{~h}$ light:dark cycle. elavGal4[C155] and UAS-mCD8GFP were obtained from the Bloomington Drosophila Stock Center. The Lamin RNAi hairpins (107419 and 45635) and $w^{1118}$ were obtained from the Vienna Drosophila Resource Centre's knockdown libraries (Dietzl et al., 2007). An isogenic $w^{1118}$ background was maintained throughout all experiments.

\section{Lifespan assay}

For assessment of fly lifespan, 3 vials of 15 male flies were collected per genotype and the number of dead flies was counted every 2-3 days when flies were transferred to new medium. This experimental paradigm was repeated at least three times.

\section{Climbing assay}

Locomotor function was assessed once per week throughout the fly's lifespan, as previously described [33, 34]. Briefly, flies were gently tapped to the bottom of the vial and given 10 seconds to climb a distance of 5 centimeters. The number of flies that failed to reach the distance was then recorded. Each vial was tested three times per time point with 30 seconds rest in between trials.

\section{qRT-PCR for knockdown efficiency}

RNA was extracted from $\sim 30$ heads of 10-day-old male flies using Purezol (Bio-Rad Laboratories). All extracted RNA samples met the quality for GPCR (A260/A280>2.0, A260/A230>1.8). After quantification, $1 \mu \mathrm{g}$ of RNA was reverse transcribed into CDNA using the iScript cDNA synthesis kit (Bio-Rad Laboratories). qRT-PCR was performed using a Sensimix probe and Syto 9 dye as per the manufacturer's instructions (Bioline) and run on a LightCycler 480 Instrument II (Roche). Cycling conditions were as follows:

1. Initial denaturation: $95^{\circ} \mathrm{C}$ for 10 minutes
2. PCR cycling: $95^{\circ} \mathrm{C}$ for 15 seconds, $55^{\circ} \mathrm{C}$ for 15 seconds, $72^{\circ} \mathrm{C}$ for 15 seconds

$$
>\text { Repeat for } 45 \text { cycles }
$$

3. Melting curve analysis

Gene expression was normalised to the reference gene $r p 49$ and relative mRNA abundance was calculated by the $\triangle \Delta C T$ method. Primers were designed for Lamin (forward: CCACTGCTGAGGGCAATGTC; reverse: TTCCAGGTTCTTCCGCGTTT) and rp49 (forward: CGGATCGATATGCTAAGCTGT; reverse: GCGCTTGTTCGATCCGTA) using PrimerBlast (NIH).

\section{Western blot}

30 Drosophila heads from 10-day-old male flies were homogenised for each genotype using a motorised pestle in $60 \mu \mathrm{L}$ of $1 \times$ Laemmli loading buffer. Homogenised samples were then boiled at $100^{\circ} \mathrm{C}$ for 5 minutes and loaded onto a $10 \%$ SDS-page gel. Proteins were separated by electrophoresis at a constant voltage of $120 \mathrm{~V}$ for 90 minutes. Precision Plus Protein WesternC pre-stained molecular weight standards (Bio-Rad Laboratories) were loaded onto the gels for protein size comparisons. Gels were transferred onto a PVDF (Bio-Rad Laboratories) membrane at $1.0 \mathrm{~A}, 25 \mathrm{~V}$ for 30 minutes, using the Trans-Blot Turbo system (Bio-Rad Laboratories). Immunoblots were blocked in $5 \%$ non-fat milk in TBS $(0.8 \% \mathrm{NaCl}, 0.2 \%$ Tris, $\mathrm{pH}$ 7.6) for 30 minutes and then incubated with $\beta$-actin (ab8227; 1:2000; Abcam) and Lamin (\#AL67.10; 1:50; Developmental Studies Hybridoma Bank) primary antibodies for 24 hours at $4^{\circ} \mathrm{C}$. Blots were then washed 3 times with TBST $(0.8 \% \mathrm{NaCl}$, $0.2 \%$ Tris, $0.1 \%$ Tween-20) and incubated with the appropriate secondary antibodies for 1 hour at room temperature. Washing steps were then repeated before blot development and exposure on a Chemidoc XRS+ (Bio-Rad Laboratories) for visualisation. ImageLab software (Bio-Rad Laboratories) was used for band molecular weight and densitometry analysis.

Brain dissection and dopaminergic neuron staining and quantification

Adult brains from male flies were dissected and stained for tyrosine hydroxylase (TH) as previously described [35]. Briefly, brains were dissected in ice-cold $\mathrm{HL}-3$ solution $(70 \mathrm{mM} \mathrm{NaCl}$, $5 \mathrm{mM} \mathrm{KCl}, 10 \mathrm{mM} \mathrm{NaHCO}, 5 \mathrm{mM}$ HEPES, $115 \mathrm{mM}$ sucrose, 5 $\mathrm{mM}$ trehalose, $20 \mathrm{mM} \mathrm{MgCl}$, $\mathrm{pH}$ 7.2) and fixed in $4 \%$ paraformaldehyde (PFA)/ phosphate-buffered saline with $0.4 \%$ Triton X-100 (PBT) solution for 20 minutes at room temperature. Fixed brains were then washed twice with quick inversion and then three times for 20 minutes at room temperature, using PBT. Brains were blocked with $5 \%$ normal goat serum in PBT for 30 minutes at room temperature. Blocked brains were then stained with primary antibodies; anti-TH (1:100; Millipore \#AB152) and anti-nc82 (1:75; Developmental Studies Hybridoma Bank) for two nights at $4^{\circ} \mathrm{C}$ and then washed as above. The secondary antibodies goat anti-mouse Alexa 555 and goat anti-rabbit Alexa 647 (1:500; Invitrogen Molecular Probes) were then applied for two nights at $4^{\circ} \mathrm{C}$. Stained and dissected brains were then washed again and mounted in Vectashield (Vector Laboratories) as previously described [27]. Confocal stacks of brains were acquired using a Leica SP8X confocal microscope and used to quantify the number of dopaminergic neurons present in the PAM, PAL, PPL1, PPL2, PPL3, PPM1, PPM2 clusters. The number of neurons was scored off blinded z-stacks using the ImageJ Cell Counter plugin. Maximum pro- 
jections of the posterior and anterior orientations were also collected.

\section{Electrophysiological recordings from the GFS}

GFS recordings were performed as described previously [19]. Briefly, flies were anesthetised on ice and then transferred to a petri dish where fly wings and legs were mounted in dental wax, ventral side down. For stimulations and recordings from the TTM and DLM, five sharp tungsten electrodes were used: two for stimulating the GF, one as a reference electrode and two for recording from the TTM and DLM, respectively. High frequency train stimulations of 20 pulses were delivered to the GF at 50, 100 and $150 \mathrm{~Hz}$. This process was repeated 10 times with 3-5 min of rest between stimulation trains. $0.2 \mathrm{~Hz}$ stimulations were used prior to each high frequency stimulation train to confirm that electrodes were still situated in the correct muscle. The probability of response, at a particular frequency of GF stimulation, due to a particular stimulus was calculated from the proportion of successful responses for both TTM and DLM pathways. Response latency was measured as the time between the stimulus artefact and the first detectable voltage deflection of the corresponding response. Latency fold change was calculated as the ratio of the $20^{\text {th }}$ pulse latency and the $1^{\text {st }}$ pulse latency of the stimulation train.

\section{Statistical methods}

Data are represented as mean \pm SEM. Climbing ability was analysed using one-way ANOVA with post-hoc Dunnett test or multiple t-tests with Holdam-Sidak correction. Lifespan was analysed using the log-rank Mantel-Cox test. GPCR and western blot data were analysed using one-way ANOVA with posthoc Dunnett test. All other experiments were analysed using student's $t$-test or two-way ANOVA with Bonferroni multiple comparisons test. Significance was considered at a $p$-value less

\section{REFERENCES}

1. López-Otín C, Blasco MA, Partridge L, Serrano M, and Kroemer $G$ (2013). The hallmarks of aging. Cell 153(6): 1194-217. doi: 10.1016/j.cell.2013.05.039

2. Burke B, and Stewart CL (2013). The nuclear lamins: Flexibility in function. Nat Rev Mol Cell Biol 14(1): 13-24. doi: 10.1038/nrm3488

3. Scaffidi P, and Misteli T (2006). Lamin A-dependent nuclear defects in human aging. Science 312(5776): 1059-63. doi: 10.1126/science. 1127168

4. Young SG, Jung $\mathrm{HJ}$, Lee JM, and Fong LG (2014). Nuclear lamins and neurobiology. Mol Cell Biol 34(15): 2776-2785. doi: 10.1128/MCB.00486-14

5. Melcer S, Gruenbaum Y, and Krohne G (2007). Invertebrate lamins. Exp Cell Res 313(10): 2157-2166. doi: 10.1016/j.yexcr.2007.03.004

6. Stuurman N, Heins S, and Aebi U (1998). Nuclear Lamins: Their Structure, Assembly, and Interactions. J Struct Biol 122(1-2): 42-66. doi: 10.1006/jsbi.1998.3987

7. Osouda S, Nakamura Y, De Saint Phalle B, McConnell M, Horigome T, Sugiyama S, Fisher PA, and Furukawa K (2005). Null mutants of Drosophila B-type lamin Dm0 show aberrant tissue differentiation rather than obvious nuclear shape distortion or specific defects during cell proliferation. Dev Biol 284(1): 219-232. doi: 10.1016/j.ydbio.2005.05.022

8. Lenz-Böhme B, Wismar J, Fuchs S, Reifegerste R, Buchner E, Betz H, than or equal to 0.05 . All statistical analysis was performed using GraphPad Prism 7.0.

\section{ACKNOWLEDGEMENTS}

We would like to thank the VDRC and Bloomington stock centers for providing an essential service to the fly community. This work was supported in part through NHMRC project grants APP1026310, APP1029672, APP1028887, APP1046090, APP1042416, APP1086851. G.G.N. is supported by an NHMRC career development fellowship II CDF1111940.

\section{SUPPLEMENTAL MATERIAL}

All supplemental data for this article are available online at www.cell-stress.com.

\section{CONFLICT OF INTEREST}

None declared.

\section{COPYRIGHT}

(C) 2018 Oyston et al. This is an open-access article released under the terms of the Creative Commons Attribution (CC BY) license, which allows the unrestricted use, distribution, and reproduction in any medium, provided the original author and source are acknowledged.

Please cite this article as: Lisa J. Oyston, Yong Qi Lin, Thang M. Khuong, Qiao-Ping Wang, Man Tat Lau, Teleri Clark and G. Gregory Neely (2018). Neuronal Lamin regulates motor circuit integrity and controls motor function and lifespan. Cell Stress 2(9): 225-232. doi: $10.15698 /$ cst2018.09.152

and Schmitt B (1997). Insertional mutation of the Drosophila nuclear lamin Dm0 gene results in defective nuclear envelopes, clustering of nuclear pore complexes, and accumulation of annulate lamellae. J Cell Biol 137(5): 1001-16. PMID: 9166402

9. Coffinier C, Jung H-J, Nobumori C, Chang S, Tu Y, Barnes RH, Yoshinaga $Y$, de Jong PJ, Vergnes L, Reue K, Fong LG, and Young SG (2011). Deficiencies in lamin B1 and lamin B2 cause neurodevelopmental defects and distinct nuclear shape abnormalities in neurons. Mol Biol Cell 22(23): 4683-4693. doi: 10.1091/mbc.E1106-0504

10. Tran JR, Chen $H$, Zheng $X$, and Zheng $Y$ (2016). Lamin in inflammation and aging. Curr Opin Cell Biol 40: 124-130. doi: 10.1016/j.ceb.2016.03.004

11. Frost B, Bardai FH, and Feany MB (2016). Lamin Dysfunction Mediates Neurodegeneration in Tauopathies. Curr Biol 26(1): 129136. doi: 10.1016/j.cub.2015.11.039

12. Dietzl G, Chen D, Schnorrer F, Su K-C, Barinova Y, Fellner M, Gasser B, Kinsey K, Oppel S, Scheiblauer S, Couto A, Marra V, Keleman K, and Dickson BJ (2007). A genome-wide transgenic RNAi library for conditional gene inactivation in Drosophila. Nature 448(7150): 151-6. doi: 10.1038/nature05954

13. Beninger RJ (1983). The role of dopamine in locomotor activity and learning. Brain Res 287(2): 173-96. PMID: 6357357

14. Mao Z, and Davis RL (2009). Eight different types of dopaminergic 
neurons innervate the Drosophila mushroom body neuropil: anatomical and physiological heterogeneity. Front Neural Circuits 3: 5 doi: 10.3389/neuro.04.005.2009

15. White KE, Humphrey DM, Hirth F, Sweeney ST, Birman S, and De $\mathrm{CN}$ (2010). The dopaminergic system in the aging brain of Drosophila. Front Neurosci 4: 205. doi: 10.3389/fnins.2010.00205

16. Seidler RD, Bernard JA, Burutolu TB, Fling BW, Gordon MT, Gwin JT Kwak Y, and Lipps DB (2010). Motor control and Aging: Links to agerelated brain structural, functional and biomechanical effects. Neurosci Biobehav Rev 34(5): 721-733. doi: 10.1016/j.neubiorev.2009.10.005

17. Riemensperger $T$, Issa $A R$, Pech $U$, Coulom $H$, Nguyễn $M V$, Cassar $M$, Jacquet $M$, Fiala $A$, and Birman $S$ (2013). A Single Dopamine Pathway Underlies Progressive Locomotor Deficits in a Drosophila Model of Parkinson Disease. Cell Rep 5(4): 952-960. doi: 10.1016/j.celrep.2013.10.032

18. Martinez VG, Javadi CS, Ngo E, Ngo L, Lagow RD, and Zhang $B$ (2007). Age-related changes in climbing behavior and neural circuit physiology in Drosophila. Dev Neurobiol 67(6): 778-91. doi: 10.1002/dneu.20388

19. Tanouye MA, and Wyman RJ (1980). Motor outputs of giant nerve fiber in Drosophila. J Neurophysiol 44(2): 405-21. doi: 10.1152/jn.1980.44.2.405

20. Allen MJ, Godenschwege TA, Tanouye MA, and Phelan P (2006). Making an escape: Development and function of the Drosophila giant fibre system. Semin Cell Dev Biol 17(1): 31-41. doi: 10.1016/j.semcdb.2005.11.011

21. He $\mathrm{Y}$, and Jasper $\mathrm{H}$ (2014). Studying aging in Drosophila. Methods 68(1): 129-133. doi: 10.1016/j.ymeth.2014.04.008

22. Jones MA, and Grotewiel M (2011). Drosophila as a model for agerelated impairment in locomotor and other behaviors. Exp Gerontol 46(5): 320-325. doi: 10.1016/j.exger.2010.08.012

23. Muñoz-Alarcón A, Pavlovic M, Wismar J, Schmitt B, Eriksson M, Kylsten P, and Dushay MS (2007). Characterization of lamin Mutation Phenotypes in Drosophila and Comparison to Human Laminopathies. PLoS One 2(6): e532. doi: 10.1371/journal. pone.0000532

24. Feany $M B$, and Bender WW (2000). A Drosophila model of Parkinson's disease. Nature 404(6776): 394-8. doi: 10.1038/35006074

25. Auluck PK, and Bonini NM (2002). Pharmacological prevention of Parkinson disease in Drosophila. Nat Med 8(11): 1185-1186. doi: 10.1038/nm1102-1185

26. Trinh K, Moore K, Wes PD, Muchowski PJ, Dey J, Andrews L, and
Pallanck L (2008). Induction of the Phase II Detoxification Pathway Suppresses Neuron Loss in Drosophila Models of Parkinson 's Disease. J Neurosci 28(2): 465-472. doi: 10.1523/JNEUROSCI.4778-07.2008

27. Barone MC, and Bohmann D (2013). Assessing neurodegenerative phenotypes in Drosophila dopaminergic neurons by climbing assays and whole brain immunostaining. J Vis Exp (74): e50339. doi: $10.3791 / 50339$

28. Butler EK, Voigt A, Lutz AK, Toegel JP, Gerhardt E, Karsten P, Falkenburger B, Reinartz A, Winklhofer KF, and Schulz JB (2012). The mitochondrial chaperone protein TRAP1 mitigates $\alpha$-Synuclein toxicity. PLoS Genet 8(2): e1002488. doi: 10.1371/journal.pgen.1002488

29. De Rose F, Marotta R, Talani G, Catelani T, Solari P, Poddighe S, Borghero G, Marrosu F, Sanna E, Kasture S, Acquas E, and Liscia A (2017). Differential effects of phytotherapic preparations in the hSOD1 Drosophila melanogaster model of ALS. Sci Rep 7(December 2016): 41059. doi: 10.1038/srep41059

30. Khalil B, Cabirol-Pol MJ, Miguel L, Whitworth AJ, Lecourtois M, and Liévens JC (2017). Enhancing Mitofusin/Marf ameliorates neuromuscular dysfunction in Drosophila models of TDP-43 proteinopathies. Neurobiol Aging 54: 71-83. doi: 10.1016/j.neurobiolaging.2017.02.016

31. Blagburn JM, Alexopoulos H, Davies JA, and Bacon JP (1999). Null mutation in shaking-B eliminates electrical, but not chemical, synapses in the Drosophila giant fiber system: a structural study. J Comp Neurol 404(4): $\quad 449-58 . \quad$ doi: 10.1002/(sici)10969861(19990222)404:4<449::aid-cne3>3.3.co;2-4

32. Speese SD, Ashley J, Jokhi V, Nunnari J, Barria R, Li Y, Ataman B, Koon A, Chang YT, Li Q, Moore MJ, and Budnik V (2012). Nuclear envelope budding enables large ribonucleoprotein particle export during synaptic Wnt signaling. Cell 149(4): 832-846. doi: 10.1016/j.cell.2012.03.032

33. Ganetzky B, and Flanagan JR (1978). On the relationship between senescence and age-related changes in two wild-type strains of Drosophila melanogaster. Exp Gerontol 13(3-4): 189-196. doi: 10.1016/0531-5565(78)90012-8

34. Le Bourg E, and Lints FA (1992). Hypergravity and aging in Drosophila melanogaster. 4. Climbing activity. Gerontology 38(1-2): 59-64. doi: 10.1159/000213307

35. Wu JS, and Luo L (2006). A protocol for dissecting Drosophila melanogaster brains for live imaging or immunostaining. Nat Protoc 1(4): 2110-2115. doi: 10.1038/nprot.2006.336 